\title{
Le Droit Suisse des Sociétés en 2017 : Modifications Législatives, Jurisprudence et Doctrine
}

\author{
Damiano Canapa / Marleen Botterbrodt / Antoine Schneebeli *
}

La contribution présente, sous une forme synthétique, les principales modifications législatives, entrées en vigueur et en cours, la jurisprudence ainsi que la doctrine publiée en droit suisse des sociétés en 2017. Les modifications législatives (I.) accordent une large place à la présentation de la révision projetée du droit de la société anonyme, tout en s'intéressant également à d'autres thèmes, tel que l'initiative populaire " Entreprises responsables - pour protéger l'être humain et l'environnement ». Les résumés de jurisprudence sont classés par forme de société et décrivent en premier lieu les arrêts publiés (II.). Finalement, la doctrine est présentée en fonction du thème traité (III.).

Introduction 298

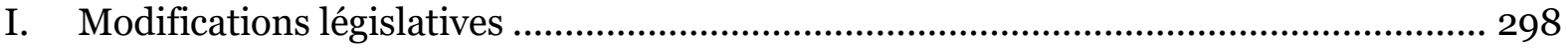

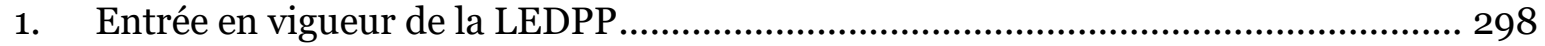

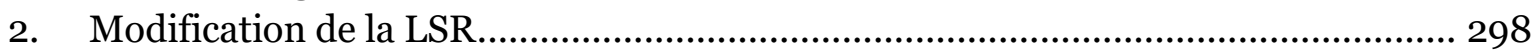

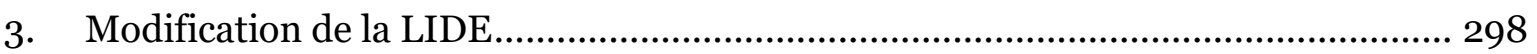

4. Modification du Code des obligations (droit du registre du commerce) ....................299

5. Modification du Codes des obligations (droit de la société anonyme) .......................299

6. L'initiative populaire « Entreprises responsables - pour protéger l'être

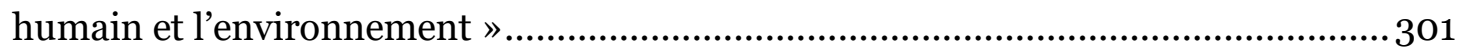

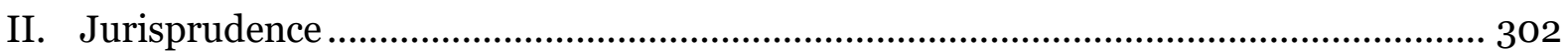

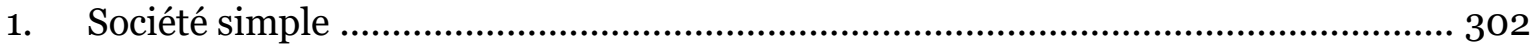

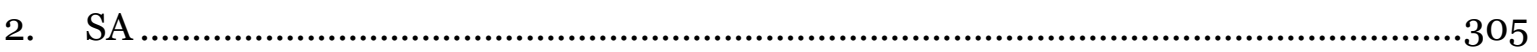

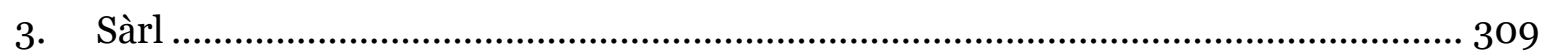

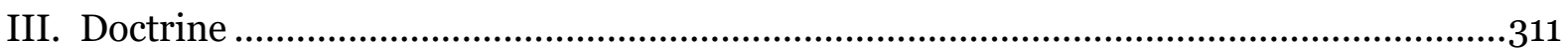

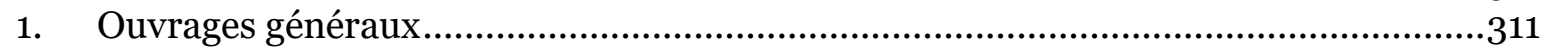

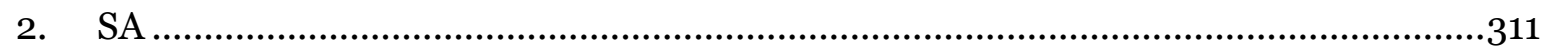

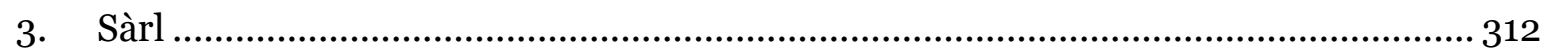

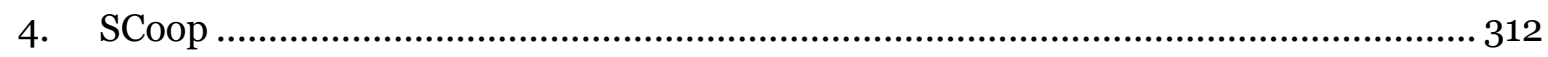

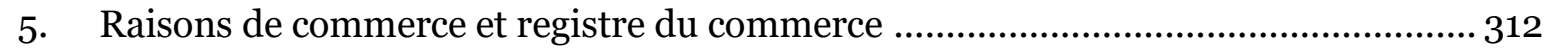

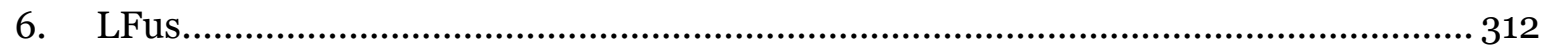

Citation : $\quad$ Damiano Canapa/Marleen Botterbrodt/Antoine Schneebeli, Le Droit Suisse des Sociétés en 2017 : Modifications Législatives, Jurisprudence et Doctrine, in: sui-generis 2018, S. 297

URL : $\quad$ sui-generis.ch/75

DOI : $\quad$ https://doi.org/10.21257/sg.75

* Damiano Canapa (damiano.canapa[at]unil.ch), Professeur à l'Université de Lausanne, LL.M. (Bruges), LL.M. (Yale) ; Marleen Botterbrodt (marleen_botterbrodt[at]hotmail.com), MLaw, Spécialiste en gestion des risques chez Maus Frères SA ; Antoine Schneebeli (antoine.schneebeli[at]unil.ch), MLaw, Assistant doctorant à l'Université de Lausanne. Les auteurs remercient MM. Cédric Ballenegger et Baptiste Allimann pour la relecture attentive de leur contribution.

$\mathrm{Ce}(\mathrm{tte})$ œuvre est mise à disposition selon les termes de la Licence Creative Commons Attribution Partage dans les Mêmes Conditions 4.0 International. 


\section{Introduction}

1 Cette contribution propose un aperçu des principales modifications législatives, entrées en vigueur et en cours, de la jurisprudence du Tribunal fédéral $[\mathrm{TF}]$ et de la doctrine publiée en matière de droit suisse des sociétés durant l'année 2017.

La section consacrée aux modifications législatives (I.) présente, en premier lieu, les textes entrés en vigueur, avant de passer en revue les modifications déjà adoptées et les messages du Conseil fédéral sur les modifications projetées. Les résumés de jurisprudence (II.), classés par forme de société, renvoient également aux éventuels commentaires dont les arrêts ont été l'objet. Finalement, les ouvrages de doctrine (III.) sont recensés en fonction du thème traité.

\section{Modifications législatives}

\section{Entrée en vigueur de la LEDPPi}

3 Cette loi est le résultat des préoccupations, à l'échelle internationale, liées à la lutte contre l'optimisation fiscale des entreprises multinationales. Elle trouve son origine dans le plan d'action BEPS (Base Erosion and Profit Shifting) de l'OCDE, qui vise à lutter contre la réduction de la base imposable et le transfert de bénéfices dans des États prévoyant une imposition faible ou nulle².

Loi fédérale du 16 juin 2017 sur l'échange international automatique des déclarations pays par pays des groupes d'entreprises multinationales (RS 654.1; RO 2017 5907); Message du 23 novembre 2016 concernant l'approbation et la mise en œuvre de l'accord multilatéral entre autorités compétentes portant sur l'échange des déclarations pays par pays ( $\mathrm{FF} 2017$ 33).

2 Ce plan a également mené à la ratification par la Suisse de l'accord multilatéral entre autorités compétentes portant sur l'échange des déclara-
La déclaration pays par pays est en principe élaborée par la société mère du groupe d'entreprises multinationales et transmise automatiquement aux autorités fiscales nationales des États et territoires où le groupe est présent. Elle contient notamment des données par États et territoires sur la répartition mondiale des chiffres d'affaires et des impôts acquittés, ainsi que des données sur les principales activités économiques des entités qui constituent le groupe. Ces informations, destinées aux seules autorités fiscales, ne sont pas publiées.

\section{Modification de la LSR3}

4 La modification de la LSR introduit une déréglementation partielle du champ d'application extraterritorial de la surveillance. Elle concerne la révision exercée par la Suisse sur les organes de révision des émetteurs étrangers d'emprunts par obligations ainsi que sur ceux des filiales importantes des émetteurs étrangers. Des mesures d'accompagnement assurent la protection des investisseurs.

\section{Modification de la LIDE4}

5 La modification fait suite à la décision du Conseil fédéral de participer à la mise sur pied d'un système mondial d'identifi-

tions pays par pays (RO 2017 7247); Message, FF 201733 (Fn. 1).

3 Modification du 30 septembre 2016 de la Loi fédérale du 16 décembre 2005 sur l'agrément et la surveillance des réviseurs (RS 221.302), entrée en vigueur le $1^{\text {er }}$ octobre 2017 (RO 2017 4859); Message du $1^{\text {er }}$ juillet 2015 portant modification de la loi sur la surveillance de la révision (FF 2015 5237).

4 Modification du 16 juin 2017 de la Loi fédérale du 18 juin 2010 sur le numéro d'identification des entreprises (RS 431.03), entrée en vigueur le 15 octobre 2017 (RO 2017 5155) ; Message du 2 décembre 2016 concernant la révision partielle de la loi fédérale sur le numéro d'identification des entreprises (FF 2017 1). 
cation des acteurs des marchés financiers. Elle permet à l'Office fédérale de la statistique d'attribuer le Legal Entity Identifier (LEI) aux entreprises suisses qui en font la demande. Pour rappel, le $L E I$ est un numéro d'identification des entités juridiques. Ce numéro est unique et standardisé au niveau international. A terme, il vise à améliorer la qualité des données financières et à faciliter l'évaluation des risques systémiques.

\section{Modification du Code des obligations (droit du registre du commerce)5}

6 La modification du Code des obligations relative à la modernisation du droit du registre du commerce a été adoptée et le délai référendaire a expiré le 6 juillet 2017. La date de l'entrée en vigueur du texte est à ce jour inconnue. Cette révision a pour conséquence que le numéro AVS sera systématiquement utilisé en qualité d'identifiant pour les personnes physiques inscrites au RC. Ensuite, la déclaration Stampa disparaîtra en tant que justificatif supplémentaire: elle sera à l'avenir partie intégrante de l'acte constitutif ou de l'acte d'augmentation du capital. Finalement, la cession de parts sociales d'une Sàrl entre associés sera assouplie : le contrat de cession ne nécessitera plus l'incorporation des renvois aux droits et obligations statutaires si l'acquéreur est déjà associé (art. 785 al. 2 $\mathrm{P}-\mathrm{CO}$ ).

\section{Modification du Codes des obliga- tions (droit de la société anonyme) ${ }^{6}$}

7 Le projet de modification du droit de la SA se base sur le précédent projet de révision qui avait été approuvé par le Conseil fédéral le 21 décembre 2007 et abandonné pendant de nombreuses années au profit de révisions ponctuelles. Il vise à adapter le droit de la SA aux besoins actuels de l'économie et à transférer dans la loi les règles de l'ordonnance contre les rémunérations abusives dans les SA cotées en bourse (ORAb). Par ailleurs, le projet accroît la distinction entre les sociétés anonymes privées et celles dont les actions sont cotées en bourse, affaiblissant de la sorte un peu plus le principe de l'unité du droit de la société anonyme. Les modifications portent essentiellement sur les éléments suivants :

- Mise en ouvre de linitiative populaire contre les rémunérations abusives
5 Modification du 17 mars 2017 du Code des obligations (droit du registre du commerce) (FF 2017 2259).
6 Message du 23 novembre 2016 concernant la modification du code des obligations (Droit de la société anonyme) (FF 2017 353); à ce sujet, voir également : Peter Böckli, Kritischer Blick auf die Botschaft und den Entwurf zur Aktienrechtsrevision 2016, GesKR 2/2017, 133 ; Peter Forstmoser/Marcel Küchler, Die Aktienrechtsreform vor der letzten Etappe? Teil I, RSJ 4/113(2017), 73; Id., Die Aktienrechtsreform vor der letzten Etappe? Teil II, RSJ 7/113(2017), 153 ; Dieter Gericke/Andreas Müller/Daniel Häusermann/Nina Hagmann, Aktienrechtsentwurf 2016 : Verbesserungen, aber auch Schwachstellen, GesKR 1/2017, 25 ; Melanie Lehmann, Neue Haftungsordnung für Revisionsstellen - Aufhebung der Solidarität?, GesKR 3/2017, 333 ; Peter Reichart, Besserstellung der Revisionsstelle in der aktienrechtlichen Verantwortlichkeit? - Prozessrechtliche Bemerkungen zu Art. 759 Abs. 2 E-OR, RSDA 5/2017, 661; Hans Caspar von der Crone/Luca Angstmann, Kernfragen der Aktienrechtsrevision, RSDA 1/2017, 3 ; Id., Vorschläge zur Aktienrechtsrevision, RSDA 5/2017, 607. 
Le projet de révision concrétise le mandat constitutionnel de l'art. 95 al. 3 de la Constitution fédérale [Cst.] en transférant les dispositions de l'ORAb dans la loi. Certains aspects renforceront la sécurité du droit et les droits des actionnaires (interdiction et limitation de certaines primes d'embauche et indemnités pour prohibition de concurrence, simplification de la procédure relative à l'action en restitution de rémunérations et vote des actionnaires sur les rémunérations).

- Fixation de seuils pour la représentation des genres

9 Des seuils de représentation des genres seront instaurés dans les grandes sociétés cotées afin de veiller au respect de l'égalité hommes-femmes dans la vie professionnelle, prévue à l'art. 8 al. 3 Cst. En cas d'acceptation du projet, les directions et les conseils d'administration devront compter respectivement $20 \%$ et $30 \%$ de femmes après des délais de transition de dix et cinq ans. Si les seuils ne sont pas atteints, le rapport de rémunération devra en exposer les raisons et présenter des mesures pour y pallier (principe comply or explain).

- Transparence dans les sociétés extractrices de matières premières 7

Le projet a pour but de renforcer la transparence des échanges financiers dans le secteur des sociétés extractrices de matières premières, afin de responsa-

\footnotetext{
A ce sujet, voir également: Lukas Handschin, Konzernverantwortungsinitiative : Gesellschaftsrechtliche Aspekte, PJA 8/2017, 998 ; Evelyne Schmid, Exigences internationales de prendre des mesures législatives, PJA 8/2017, 930.
}

biliser celles-ci et de remédier à l'insuffisance de cadre légal dans certains pays. Un rapport, publié par voie électronique, devra déclarer les paiements supérieurs à fr. 100'000 par année effectués au profit de gouvernements.

- Flexibilité des dispositions sur la fondation et sur le capital

11 L'utilisation d'une monnaie étrangère pour la fixation du capital-actions et la réduction autorisée de celui-ci - à travers l'introduction d'une «marge de fluctuation du capital », qui permettra à l'assemblée générale [AG] d'autoriser le conseil d'administration à augmenter ou réduire le capital-actions dans certaines limites durant un délai de maximum cinq ans (art. 653s ss P-CO) ${ }^{8}$ - seront toutes deux autorisées. La valeur nominale minimale des actions d'un montant d'un centime disparaitra au profit d'une "valeur nominale supérieure à zéro centime ». La forme authentique ne sera plus nécessaire pour la fondation, la radiation et la dissolution du registre du commerce d'une SCoop, d'une Sàrl ou d'une SA, lorsque les statuts se contentent du minimum légal, que le capital-actions ou le capital social est fixé en francs, et que les apports sont entièrement libérés en francs. Les statuts d'une telle société pourront également être modifiés sans recours à un officier public, sauf en cas de réduction du capital-actions ou du capital social, ou dans le cas d'une augmentation du capital-actions ou du capital social effectuée autrement qu'uniquement en

8 A ce sujet, voir également: Olivier Baum, Kapitalband und bedingtes Kapital im Entwurf zur Aktienrechtsrevision, GesKR 1/2017, 47 ; Hans Caspar von der Crone/Linus Cathomas, Das Aktienkapital im Entwurf zur Aktienrechtsrevision, RSDA 5/2017, 586. 
francs9. D’autres mesures liées à l'assainissement et à l'utilisation de tribunaux arbitraux pour certains litiges sont également à noter.

\section{- Autres modifications projetées}

Les possibilités de recourir aux médias électroniques en matière d'AG virtuelle seront étendues ${ }^{10}$. De plus, des modifications sont prévues en matière de gouvernance d'entreprise, qui se traduiront notamment par un abaissement de seuils pour l'exercice des droits des actionnaires et par une différenciation de ces seuils selon qu'ils s'appliqueront à une société anonyme privée ou à une société anonyme cotée en bourse; ces seuils concernent le droit à l'inscription d'un objet à l'ordre du jour, le droit de proposition, le droit de requérir la convocation de l'assemblée générale et le droit de faire ordonner un examen spécial ${ }^{11}$.

\section{L'initiative populaire « Entreprises responsables - pour protéger l'être humain et l'environnement \12 $^{12}$}

Le Conseil fédéral propose aux Chambres fédérales de soumettre au peuple et aux cantons l'initiative " Entreprises responsables - pour protéger l'être humain et l'environnement » sans contre-projet et en lui recommandant de la rejeter. Le texte, déposé le 10 octobre 2016 avec le

$9 \overline{\text { A ce sujet, voir également : Loïc Horisberger, La }}$ suppression des règles sur la reprise de biens sans allégement des règles sur les apports en nature - une incohérence, REPRAX 4/2017, 183.

10 A ce sujet, voir également : Hans Caspar von der Crone/Thomas Grob, Die virtuelle Generalversammlung, RSDA 1/2018, 5 .

11 Message, FF 2017353 (n 6), 409 sqq.

12 Message du 15 septembre 2017 relatif à l'initiative populaire « Entreprises responsables - pour protéger l'être humain et l'environnement » (FF 2017 5999). nombre requis de signatures, vise les entreprises suisses actives à l'étranger et les entreprises suisses contrôlant des entreprises étrangères. Il demande que la Confédération prenne des mesures pour garantir que ces entreprises fassent preuve d'une diligence raisonnable afin d'assurer le respect des droits humains internationalement reconnus et des normes environnementales internationales. En cas de violation de ces règles, les entreprises pourront être tenues pour responsables du dommage causé et devront en répondre, sur le plan civil, en Suisse. Dans ce cadre, le texte de l'initiative prévoit d'inverser le fardeau de la preuve : en cas de mise en cause, il appartiendra aux entreprises de prouver qu'elles ont fait preuve de toute la diligence requise par les circonstances pour assurer le respect - par elles-mêmes ou par les entreprises qu'elles contrôlent - des droits humains et des normes environnementales ${ }^{13}$.
13 L'initiative a entre-temps été débattue dans le cadre de la réforme du droit de la société anonyme et, en dépit de la position du Conseil fédéral, un contre-projet indirect à l'initiative a été adopté sur la base du « Rapport complémentaire de la Commission des affaires juridiques [du Conseil national] du 18 mai 2018 sur les propositions de la commission en vue du dépôt d'un contre-projet indirect à l'initiative populaire « Entreprises responsables - pour protéger l'être humain et l'environnement » dans le cadre de la révision du droit de la société anonyme ». La reprise de nombre des aspects de l'initiative dans le contreprojet pourrait entraîner le retrait de celle-là : Michel Guillaume, Le comité de l'initiative sur les multinationales responsables prêt à retirer son initiative, Le Temps, 12 juin 2018. 


\section{Jurisprudence}

\section{Société simple}

\section{a) Détermination de l'existence d'une société simple14}

14 Faits : Au printemps 2010, la société S1 SA s'intéresse à l'achat d'un parc d'immeubles. Elle conclut un contrat de crédit sur gage immobilier avec la banque B qui lui octroie un crédit hypothécaire de 111 millions de francs. Le contrat prévoit une obligation de versement de 10 millions de francs de $\mathrm{S} 1$ à la banque à titre de sûretés.

Le 27 avril 2010, la banque B indique par courriel que les fonds pour le prix d'achat global sont en cours de transfert. Une semaine plus tard, n'ayant pas reçu le versement, le propriétaire du parc immobilier met la banque $\mathrm{B}$ en demeure. Cette dernière reproche à $\mathrm{S} 1 \mathrm{SA}$ de ne pas avoir versé les 10 millions de francs et refuse d'agir dans l'intervalle. Le 11 mai 2010, la banque déclare mettre fin au contrat de prêt avec effet immédiat.

Saisi par S1 SA, le Tribunal de première instance du canton de Genève rejette une demande en reddition des comptes à l'encontre de la banque B. Fondé sur les dispositions de la société simple, le jugement d'appel ordonne à la banque $\mathrm{B}$ de remettre à S1 SA l'intégralité des documents reçus, créés, établis, rédigés et utilisés lors du projet. La banque B recourt auprès du TF. cipal permettant de déterminer si l'on se

14 Arrêt du Tribunal fédéral 4A_251/2016 du 13 décembre 2016. trouve en présence d'une société simple est l'animus societatis ${ }^{15}$. Dans le cas d'espèce, la banque assume principalement les risques d'une bailleuse de fonds, ce qui ne suffit pas à prouver l'existence d'une substance partagée. Malgré la présence d'une volonté réciproque d'atteindre un but commun par des efforts fournis par les deux parties, l'existence d'une société simple entre S1 SA et la banque B est niée ${ }^{16}$. La cause est renvoyée à l'autorité précédente qui devra examiner le grief selon lequel une reddition de compte pourrait se faire sur le droit du mandat (art. 400 CO) ${ }^{17}$.

\section{b) Répartition du bénéfice de liquidation d'une société simple18}

18 Faits : En octobre 2007, $\mathrm{Z}$ et les époux X créent une société simple dans le but de construire et de vendre des maisons d'habitation. L'apport des époux $\mathrm{X}$ se compose d'un terrain divisé en deux parcelles, d'une valeur globale de fr. 1'900'000. Z s'oblige à fournir, entre autres, des prestations d'architecte et à faire deux apports en espèces pour un total de fr. 850'ooo.

19 Le 19 septembre 2008, les coassociés conviennent de la liquidation de la société ainsi que de la répartition des béné-

$15 \overline{\text { Soit la volonté de mettre en commun des biens, }}$ des ressources ou des activités en vue d'atteindre un objectif déterminé, d'exercer une influence sur les décisions et de partager les risques, les bénéfices ainsi que la substance même de l'entreprise, Arrêt du Tribunal fédéral 4A_251/2016 du 13 décembre 2016, consid. 5.2.

16 Arrêt du Tribunal fédéral 4A_251/2016 du 13 décembre 2016, consid. 5.3.

17 Arrêt du Tribunal fédéral 4A_251/2016 du 13 décembre 2016, consid. 5.4.

18 Arrêt du Tribunal fédéral 4A_245/2016 du 19 décembre 2016. 
fices. Le 24 septembre 2008, les deux parcelles sont vendues par les époux X pour un montant de fr. 2'400'0oo. Le 19 décembre 2008, les époux X conviennent avec l'acheteur de finalement ne lui céder qu'une des parcelles, pour fr. 1'200'ooo, et de conserver la seconde.

20 Au terme de la procédure de poursuite intentée par $\mathrm{Z}$ en relation avec le calcul du bénéfice de la société simple, la mainlevée définitive de l'opposition est prononcée le 16 juillet 2014 par le Tribunal civil de l'arrondissement de La Côte et confirmée par le Tribunal cantonal. Les époux $\mathrm{X}$ recourent en matière civile contre ce jugement.

21 Droit: Les époux X soutiennent que la vente de la parcelle n'est pas une affaire de la société, car elle intervient après la date de sa liquidation. Les parties auraient, de plus, convenu que les époux $\mathrm{X}$ reprenaient les deux parcelles à leur valeur d'apport de fr. 1'900'ooo, sans bénéfice $^{19}$. Le TF estime lui que le prix de vente initial des parcelles de fr. 2'400'0oo doit revenir à la société et que l'opération du 19 décembre 2008 se rapportait en fait au rachat ultérieur de l'une des deux parcelles par les époux $\mathrm{X}$ au prix de fr. 1'200'ooo. Le bénéfice de la vente correspond par conséquent à la différence entre le prix de vente de fr. 2'40o'ooo et la valeur de l'apport de fr. 1'900'ooo (fr. 500'000).

Le TF rappelle que, selon l'art. 548 al. 1 et $2 \mathrm{CO}$, celui qui fait un apport en propriété ne le reprend pas en nature dans la liquidation, mais qu'il a droit au prix pour lequel son apport a été accepté.

19 Arrêt du Tribunal fédéral 4A_245/2016 du 19 décembre 2016, consid. 2.
Même si ces règles ne s'appliquent généralement pas aux immeubles, la plusvalue doit être répartie entre les associés lorsque l'activité de la société a, comme dans le cas présent s'agissant du travail de Z, augmenté la valeur du bien. Partant, le TF rejette le recours ${ }^{20}$.

\section{c) Dissolution d'une société simple en raison de l'impossibilité d'atteindre le but social21}

23 Faits : Depuis 1994, X et Z exploitent un cabinet médical sous la forme d'une société simple. La convention d'exploitation prévoit une clause de résiliation moyennant un délai d'une année en cas d'incapacité de travail pour maladie de longue durée. La relation des associés se détériore dès 2005, causant un «burnout » à X. Le 27 juillet 2006, X résilie le contrat de société simple avec effet immédiat, ce à quoi Z s'oppose.

24 X ouvre action devant la Cour civile du Tribunal cantonal vaudois. Le comportement de $\mathrm{Z}$, qui aurait provoqué l’incapacité de travail de $\mathrm{X}$, rendrait la réalisation du but social impossible. Le Tribunal cantonal retient que l'incapacité subjective de travailler ensemble ne constitue pas un motif permettant une résiliation de contrat avec effet immédiat. $\mathrm{X}$ recourt auprès du $\mathrm{TF}$.

25 Droit: Le TF passe en premier lieu en revue la controverse doctrinale relative au motif de dissolution fondé sur l'impossibilité subséquente d'atteindre le but social. Certains auteurs sont d'avis qu'il ne peut s'agir que d'une impossibili-

20 Arrêt du Tribunal fédéral 4A_245/2016 du 19 décembre 2016, consid. 3.

21 Arrêt du Tribunal fédéral 4A_426/2016 du 10 janvier 2017, consid. 3. 
té objective ${ }^{22}$. D'autres exposent qu'un associé dispose d'un droit de dénonciation sans délai fondé sur l'art. 27 al. 2 CC s'il est raisonnablement impossible d'imposer à un associé de rester dans la société; selon ces auteurs, les motifs permettant une résiliation immédiate doivent être encore plus graves et pressants que dans le cas d'une dissolution pour justes motifs (art. 545 al. $7 \mathrm{CO}$ ) ${ }^{23}$.

Sans répondre à la controverse, le $\mathrm{TF}$ indique que l'impossibilité subjective de continuer à exploiter conjointement le cabinet médical ne satisfait pas aux conditions de l'art. 545 al. 1 ch. 1 CO. Cette impossibilité ne constitue donc pas un motif pouvant entraîner la dissolution immédiate de la société : le but social de la société simple, qui est de partager les charges d'infrastructure du cabinet, reste réalisable durant le délai de résiliation d'une année en cas d'impossibilité subjective24.

Par ailleurs, $\mathrm{X}$ n'ayant pas expliqué les raisons pour lesquelles le fait de supporter les charges d'exploitation pendant un an représenterait un engagement excessif, elle ne saurait s'appuyer sur l'art. 27 al. 2 CC. X aurait également pu proposer un remplaçant qui, s'il avait accepté les termes de la convention d'exploitation, n'aurait pu être refusé par Y que dans les limites de l'art. 2 CC. Finalement, le conflit ayant été imputable aux deux parties, Y n'a pas commis d'abus de droit en refusant la résiliation immédiate ${ }^{25}$.

22 Arrêt du Tribunal fédéral 4A_426/2016 du 10 janvier 2017, consid. 3.3.2.

23 Arrêt du Tribunal fédéral 4A_426/2016 du 10 janvier 2017, consid. 3.3.3.

24 Arrêt du Tribunal fédéral 4A_426/2016 du 10 janvier 2017, consid. 3.4.

25 Arrêt du Tribunal fédéral 4A_426/2016 du 10 janvier 2017, consid. 3.5.

\section{d) Qualité pour agir en cas de contrat de société simple ${ }^{26}$}

28 Faits : A et B sont associés d'une étude d'avocats. Z utilise leurs locaux pour exercer son activité à titre d'indépendant. Un contrat de société simple règle cette collaboration. Après des dissensions, A ouvre action contre $\mathrm{Z}$ pour inexécution du contrat de société simple.

29 Le TF doit se prononcer sur la qualité pour agir de A. Celle-ci lui a été refusée par les instances inférieures au motif qu'il aurait dû agir conjointement avec B.

30 Droit : Le TF commence par rappeler que tous les membres de la société simple doivent nécessairement ouvrir action ensemble, comme consorts nécessaires (art. 70 al. $1 \mathrm{CPC}$ ) 27 . Puisque la communauté que forme la société simple découle du droit matériel (art. 544 al. 1 CO), il s'agit d'une consorité matérielle nécessaire, dont l'existence peut être librement examinée par le TF. A défaut d'action commune, il n'existe pas de qualité pour agir, ce qui entraîne le rejet de la demande ${ }^{28}$.

31 En l'espèce, $\mathrm{A}$ et $\mathrm{B}$, associés de l'étude, ont conclu conjointement le contrat de collaboration avec $\mathrm{Z}$, non associé. Partant, A et B forment une société simple (indépendante de la société simple qu'ils forment avec Z) et doivent agir conjointement contre $\mathrm{Z}$. Le TF confirme l'analyse des instances cantonales et rejette le recours ${ }^{29}$.

26 Arrêt du Tribunal fédéral 4A_217/2017 du 4 août 2017.

27 Arrêt du Tribunal fédéral 4A_217/2017 du 4 août 2017, consid. 3.3.

28 Arrêt du Tribunal fédéral 4A_217/2017 du 4 août 2017, consid. 3.4.

29 Arrêt du Tribunal fédéral 4A_217/2017 du 4 août 2017, consid. 4. 


\section{a) Voix prépondérante lors de la désignation de l'organe de révision3o}

32 Faits : Le capital social de Hôtel A SA se monte à fr. 500'000 (380 actions nominatives ordinaires à fr. 1000 et 1200 actions nominatives à droit de vote privilégié à fr. 100). C.B et D.B possèdent chacun 65 actions nominatives ordinaires et 600 actions nominatives à droit de vote privilégié (soit 665 voix et une part de capital social de fr. 125'0oo chacun). E.B possède 250 actions ordinaires (soit 250 voix et une part de capital social de fr. 250'000). G, président du conseil d'administration, n'est pas actionnaire.

33 Alors que C.B et D.B y sont favorables et que E.B s'y oppose, l'organe de révision de Hôtel A SA, H SA, est réélu dans sa fonction grâce à la voix prépondérante du président du conseil d'administration. E.B fait recours contre cette décision.

34 Droit: Le TF rappelle que les statuts peuvent déclarer que le droit de vote sera exercé proportionnellement au nombre des actions détenu par chaque actionnaire, sans égard à leur valeur nominale (art. 693 al. 1 CO). Cette réglementation n'est toutefois pas applicable lorsqu'il s'agit de désigner l'organe de révision (art. 693 al. 3 CO) : la détermination du droit de vote s'effectue alors obligatoirement de manière proportionnelle à la valeur nominale des actions ${ }^{31}$. Il est ainsi impossible, dans ce cas, d'accorder la

ATF 143 III 120.

31 ATF 143 III 120, consid. 3.1. voix prépondérante au président du conseil d'administration ${ }^{2}$.

\section{b) Engagement excessif en cas de convention d'actionnaires 33}

35 Faits : D SA est fondée le 10 janvier 1985 par A, B et C. Le 23 janvier 1985, les trois actionnaires concluent une convention d'actionnaires qui comprend, notamment, un droit de préemption, une prétention des fondateurs à siéger au conseil d'administration et une clause prévoyant que si le salaire de A, qui occupe le poste de directeur, dépasse un certain montant, un pourcentage du dépassement doit être versé à B. La convention, qui est conclue pour une durée indéterminée, ne peut pas être dénoncée et n'est modifiable qu'avec l'accord de toutes les parties. En cas de violation, une peine conventionnelle de fr. 40'0oo est prévue.

36 En décembre 1986, B quitte le conseil d'administration de D SA. En 1998, des négociations ont lieu entre les actionnaires en vue de modifier la convention d'actionnaires. Leur échec entraîne la résiliation de la convention par $\mathrm{A}$, ce à quoi B s'oppose. A partir de l'AG de 1999, B requiert chaque année son élection au conseil d'administration, sans jamais être élu.

$32 \overline{\text { ATF } 143 \text { III 120, consid. 3.2. Cet arrêt constitue }}$ donc un changement de pratique, dans le sens d'une restriction, en matière d'admissibilité de la voix prépondérante du président du conseil d'administration en cas d'égalité de voix de l'assemblée générale. S'agissant de l'ancienne pratique, voir également : Dubs/Truffer, art. 703, in : Honsell/Vogt/Watter (édits.), Basler Kommentar, Obligationenecht II, 5 ième éd., Bâle 2016, N 12-16 (cité : BSK OR II-Auteure).

33 ATF 143 III 480; à ce sujet, voir également: Fabiano Menghini, Aktionärbindungsvertrag: Übermässige Bindung und deren Folgen, GesKR 3/2017, 355 ; Patricia Reichmuth/Hans Caspar von der Crone, Übermässige Bindung im Aktionärbindungsvertrag, RSDA 5/2017, 703. 
devant le Kantonsgericht du Canton d'Appenzell Rhodes-Extérieures. Il requiert fr. 160'ooo plus intérêts suite aux violations répétées de la convention d'actionnaires. Au surplus, A doit être tenu d'élire $B$ au conseil d'administration lors de la prochaine AG de D SA et de le réélire aussi longtemps qu'il le souhaitera. À l'exception du dernier point, le Kantonsgericht donne entièrement raison au demandeur. L'Obergericht du même canton confirme le jugement. A recourt au TF.

Droit : S'exprimant sur le droit de refuser l'exécution d'un contrat sur la base de l'art. 27 al. 2 CC, le TF indique que cet article concerne un cas de résiliation d'un contrat de durée pour engagement excessif, et non pour juste motif. Un engagement excessif découle, avant tout, de la conception du contrat, en combinaison avec la durée de ce dernier. Un juste motif menant à la résiliation d'un contrat de durée découle, quant à lui, d'une modification des bases contractuelles objectives ou des relations personnelles d'une partie au contrat. Le but de l'art. 27 al. 2 CC exige ainsi que la partie engagée de manière excessive puisse refuser son exécution, sans avoir à résilier le contrat. Un engagement excessif n'est pas constaté d'office par l'autorité, sauf dans le cas où un droit strictement personnel est touché34.

Le TF juge qu'un engagement de longue durée lié à la qualité d'actionnaire est admissible s'il peut être renoncé à cet engagement à des conditions n'étant pas excessivement contraignantes. Les circonstances au moment de l'exercice de la

$34 \widehat{\text { ATF } 143 \text { III 480, consid. 4.2. }}$ prétention sont déterminantes 35 . Un tel engagement est néanmoins excessif lorsqu'il fait obstacle à un plan de succession d'entreprise, en affectant le libre exercice de l'activité économique d'une partie et en empiétant, du coup, sur son domaine d'activité personnel. Tel est le cas ici, car, selon les termes de la convention d'actionnaires, un nouvel actionnaire devrait obligatoirement souscrire à la convention et qu'il serait par conséquent restreint par le droit de préemption et par l'obligation, s'il occupait le poste de directeur, de verser, potentiellement, une partie de son salaire à $\mathrm{B}$. La convention d'actionnaires, plus de trente ans après sa conclusion, doit donc être limitée temporellement et être déclarée caduque avec effet ex nunc ${ }^{36}$.

\section{c) Responsabilité du conseil d'administration de la SA dans la liquidation 37}

40 Faits : Une procédure de faillite est ouverte à l'encontre de $\mathrm{D} \mathrm{SA}$, qui est régie par l'administrateur unique A. La procédure se termine faute d'actifs et D SA est radiée du registre du commerce en juin 2009.

41 En juin 2010, les créanciers de la société, B et C, se basant sur les art. 757 al. 2 cum $754 \mathrm{CO}$, actionnent A en réparation du dommage subi par la violation de son devoir dans l'administration, la gestion et la liquidation de D SA. Suite à l'admission de l'action par l'Obergericht du Canton de Thurgovie, A recours au TF.

35 ATF 143 III 480, consid. 5.

36 ATF 143 III 480, consid. 5.6.2.

37 Arrêt du Tribunal fédéral 4A_384/2016 du $1^{\mathrm{er}}$ février 2017. 

et $2 \mathrm{CO}$, il existe, dès l'ouverture de la faillite, une créance unique de la communauté des créanciers qui ne peut être exercée qu'après que l'administration de la faillite a renoncé à faire valoir cette créance. Chaque créancier peut alors agir à la place de l'administration et invoquer la créance de la communauté. Le mécanisme correspond à celui de l'art. 260 LP et peut aussi être exercé lorsque la société faillie est radiée du registre du commerce: un créancier social peut alors demander la réinscription de la société en vue d'obtenir de la communauté des créanciers la cession du droit d'agir contre les administrateurs 38 .

43 Dans le cas d'espèce, sans la réinscription de D SA au registre du commerce et sans procédure de collocation, $\mathrm{B}$ et $\mathrm{C}$ n'étaient pas légitimés à agir et à faire valoir leurs créances sur la base de l'art. 757 al. 2 CO39. Partant, le recours est fondé40.

\section{d) Carence dans l'organisation de la SA41}

44 Faits : $\mathrm{A}$ et $\mathrm{B}$ sont actionnaires, à 50\% chacun, de la société X SA. En raison d'un conflit persistant entre les deux actionnaires, l'AG ne parvient plus à prendre les décisions relevant de sa compétence. Le 19 janvier 2015, le Tribunal de première instance du Canton de Genève désigne U en qualité d'administrateur de $\mathrm{X}$ SA. B s'oppose à la nomination et demande qu'A lui cède la totalité de ses ac-

$38 \overline{\text { Arrêt du Tribunal fédéral 4A_384/2016 du } 1^{\mathrm{er}}}$ février 2017, consid. 2.1.

39 Arrêt du Tribunal fédéral 4A_384/2016 du $1^{\text {er }}$ février 2017, consid. 2.3.

40 Arrêt du Tribunal fédéral 4A_384/2016 du $1^{\mathrm{er}}$ février 2017, consid. 2.4.

41 Arrêt du Tribunal fédéral 4A_51/2017 du 30 mai 2017. tions. A refuse et requiert, dans sa demande reconventionnelle, la dissolution de X SA et sa liquidation.

45 Le Tribunal ordonne la vente aux enchères des actions de $\mathrm{X}$ SA et que seuls $\mathrm{A}$ et $\mathrm{B}$ pourront enchérir. $\mathrm{B}$ fait appel devant la Chambre civile de la Cour de justice qui confirme le jugement. B recourt devant le TF.

46 Droit : En cas de carence dans l'organisation d'une SA, l'art. $731 b$ al. 1 CO requiert du tribunal qu'il prenne les mesures nécessaires, notamment la nomination de l'organe qui fait défaut ou d'un commissaire, ou la dissolution et la liquidation de la société selon les règles de la faillite. Le juge peut envisager d'autres mesures, mais il doit respecter le principe de proportionnalité. La demande reconventionnelle de A de dissoudre la société est, par conséquent, une mesure de dernier recours.

47 Le $\mathrm{TF}$ admet la vente aux enchères des actions entre $\mathrm{A}$ et $\mathrm{B}^{42}$. Il retient que les actionnaires doivent être traités de manière égale et que $B$ ne peut pas exiger un droit préférentiel à l'acquisition des actions. La procédure a pour but d'aboutir à une résolution rapide du blocage, mais ne vise pas à résoudre le conflit entre actionnaires43. S'agissant de l'administrateur U, la Haute Cour constate que le juge a le droit de nommer un commissaire et de prolonger sa mission, même si les actionnaires sont insatisfaits de la personne du fait d'une coopération insuffisante44. Le recours est donc rejeté45.

42 Arrêt du Tribunal fédéral 4A_51/2017 du 30 mai 2017, consid. 5.

43 Arrêt du Tribunal fédéral 4A_51/2017 du 30 mai 2017, consid. 6.

44 Arrêt du Tribunal fédéral 4A_51/2017 du 30 mai 2017, consid. 7. 


\section{e) Prise de décision en cas d'actions détenues en copropriété ; conso- rité nécessaire en cas d'action ${ }^{46}$}

48 Faits : Entre 1992 et 2012, B et sa sœur A héritent de 50 actions au porteur de deux SA. A devient propriétaire de 25 actions dans chacune des deux sociétés. Les 25 actions restantes des deux sociétés, objet d'un litige successoral, restent en indivision et en propriété commune de A et B.

49 Le 27 mai 2014, les SA convoquent et tiennent leurs AG ordinaires. Selon la feuille de présence, qui indique une répartition de 37,5 actions pour $\mathrm{A}$ et de 12,5 actions pour $\mathrm{B}$, des décisions contestées par ce dernier sont approuvées. A soutient que les actions en indivision ont fait l'objet d'un partage, à raison de 12,5 actions pour chacun des héritiers, alors que le B soutient que les actions demeurent en indivision.

50 B introduit des actions en annulation et en constatation de la nullité des décisions des AG, qui sont admises par les deux premières instances.

Les deux sociétés recourent devant le TF qui doit se prononcer sur la qualité pour agir de $\mathrm{B}$ et sur la validité des décisions des AG au regard de l'art. 690 al. 1 CO.

Droit : S'agissant de la convocation des héritiers aux AG, le TF note que B contestait, depuis 2004, la conformité de la répartition des actions détenues en main commune. Par conséquent, il ne peut s'opposer, de bonne foi, à un vice affec-

45 Arrêt du Tribunal fédéral 4A_51/2017 du 30 mai 2017, consid. 9.

46 Arrêt du Tribunal fédéral 4A_516/2016 du 28 août 2017; à ce sujet, voir également : Olivia Wipf/Hans Caspar von der Crone, Aktien im Gesamteigentum, RSDA 1/2018, 83. tant sa convocation. Il lui appartenait de faire le nécessaire pour obtenir la désignation d'un représentant commun, comme le prescrit l'art. 690 al. 1 CO47.

53 À défaut de représentant commun, les actions ne peuvent de plus pas être représentées au sens de l'art. 703 CO. Par conséquent, les droits de participation et de vote liés aux actions en indivision ne peuvent pas être exercés. Pour le TF, les décisions ont été prises de manière valable, à l'unanimité, par l'intermédiaire des 25 actions propriété de $\mathrm{A}^{48}$.

54 Par ailleurs, la qualité pour agir de B lui est refusée. En l'absence d'un représentant commun pour les actions en indivision, B ne pouvait pas agir seul sur la base de l'art. $706 \mathrm{CO}$, mais il devait agir en commun avec A, comme consort matériel nécessaire (70 al. $1 \mathrm{CPC}$ ) 49 . Le recours de A est admis50.

\section{f) Contrôle spécial ; accès aux documents d'une filiale de la so- ciété faisant l'objet du contrôle51}

55 Faits : A SA est une société holding dont le capital-actions est détenu par B.B et son frère C.B à hauteur de $45 \%$ chacun, tandis que le $10 \%$ restant est détenu par D.B. A SA détient notamment la société $\mathrm{E}$ SA à $100 \%$.

47 Arrêt du Tribunal fédéral 4A_516/2016 du 28 août 2017, consid. 7.1.

48 Arrêt du Tribunal fédéral 4A_516/2016 du 28 août 2017, consid. 7.2.

49 Arrêt du Tribunal fédéral 4A_516/2016 du 28 août 2017, consid. 8.

50 Arrêt du Tribunal fédéral 4A_516/2016 du 28 août 2017, consid. 9.

51 Arrêt du Tribunal fédéral 4A_180/2017 du 31 octobre 2017. 

B.B soumet au conseil d'administration plusieurs questions concernant le groupe sans obtenir de réponse. Lors de l'AG du 14 janvier 2016, l'institution d'un contrôle spécial, proposée par B.B, est refusée par $55 \%$ des voix.

actionne A SA devant l'Obergericht du Canton de Zoug et requiert la désignation d'un contrôleur spécial afin de clarifier des questions liées à la situation financière de E SA. Cette action est partiellement admise. A SA recourt alors en matière civile.

Droit : Le TF est d'avis que la situation financière d'une filiale peut être examinée dans le cadre d'un contrôle spécial de la société mère. Il n'est partant pas exclu de requérir de la société mère la transmission de documents de sa filiale qui se trouvent en possession de cette dernière. Une telle requête n'est possible qu'à condition que les transactions commerciales de la filiale devant être clarifiées aient une incidence sur la situation financière de la société mère. Ceci n'est, par exemple, pas le cas lorsque l'atteinte à la filiale a une importance minime pour la société mère ${ }^{2}$. L'organisation de la direction et du contrôle de la filiale doit, par ailleurs, permettre à la société mère d'accéder aux documents qui ne se trouveraient pas déjà en sa possession. Il doit finalement être rendu vraisemblable que les informations requises sont nécessaires aux actionnaires pour exercer leurs droits dans la société mère53.

52 Arrêt du Tribunal fédéral 4A_180/2017 du 31 octobre 2017, consid. 4.1.

53 Arrêt du Tribunal fédéral 4A_180/2017 du 31 octobre 2017, consid. 4.4.
59 Pour le TF, une société mère qui détient sa filiale à $100 \%$ peut accéder aux documents concernant les opérations commerciales de cette dernière. Le conseil d'administration de E SA était de plus composé des mêmes personnes que celui de sa société mère54. Le TF ajoute qu'il est sans importance que le siège des deux sociétés diffère. Finalement, le fait que $\mathrm{E}$ SA ne constitue pas le seul élément d'actif de A SA n'entraîne pas, en soi, qu'une atteinte à la filiale n'exercerait aucune influence sur la situation financière de la société mère55.

\section{Sàrl}

\section{a) Refus de l'assistance judiciaire en faveur d'une personne morale56}

60 Faits : Le $1^{\text {er }}$ décembre 2014, l'Office fédéral du registre du commerce [OFRC] prononce la dissolution de A Sàrl. Dans le même temps, A Sàrl est engagée dans une procédure de bail à loyer qui l'oppose à son bailleur et pour laquelle elle sollicite l'assistance judiciaire. Suite au rejet de sa demande d'assistance judiciaire au niveau cantonal, A Sàrl saisit le TF.

61 Droit : Le TF rappelle que, contrairement aux personnes physiques, les personnes morales ne peuvent pas bénéficier de l'assistance judiciaire. Une personne morale n'est pas indigente ou pauvre, mais insolvable ou endettée, et doit se voir imposer les conséquences découlant de la concurrence. Une exception est admise

54 Arrêt du Tribunal fédéral 4A_180/2017 du 31 octobre 2017, consid. 4.5.

55 Arrêt du Tribunal fédéral 4A_180/2017 du 31 octobre 2017, consid. 4.6.

56 Lorsque la procédure pour laquelle elle est requise ne garantit pas sa survie, ATF 143 I 328. 
quand les seuls actifs de la société sont en jeu et que ses participants économiques sont sans ressources57. Néanmoins, même dans ces circonstances, l'assistance judiciaire doit être refusée lorsque la procédure pour laquelle elle est requise ne garantit pas la survie de la personne morale 58 .

En l'espèce, la décision de l'OFRC prononçant la dissolution est définitive et un retour en arrière inconcevable59. Partant, c'est à juste titre que les instances inférieures ont refusé à A Sàrl l'assistance judiciaire ${ }^{60}$.

\section{b) Devoir de diligence des administrateurs d'une Sàrl61}

63 Faits : Les sociétés C Sàrl et D SA coopèrent à la réalisation du projet $\mathrm{H}$. En décembre 2008, A octroie un prêt à C Sàrl, que cette dernière utilise pour rembourser une dette vis-à-vis de D SA et éviter la faillite. Après que $C$ Sàrl soit tombée en faillite en août 2011, A intente une action en responsabilité contre $\mathrm{B}$, administrateur unique de C Sàrl. Il exige le remboursement du prêt octroyé à $\mathrm{C}$ Sàrl au motif qu'en décidant d'utiliser cette somme pour rembourser la dette de C Sàrl vis-àvis de D SA, B aurait violé son devoir de diligence d'administrateur. Après le rejet de son action par le Bezirksgericht et par le Kantonsgericht du Canton de Schwyz, A recourt auprès du TF.

64 Droit : Le TF examine l'obligation de diligence des associés gérants. Selon

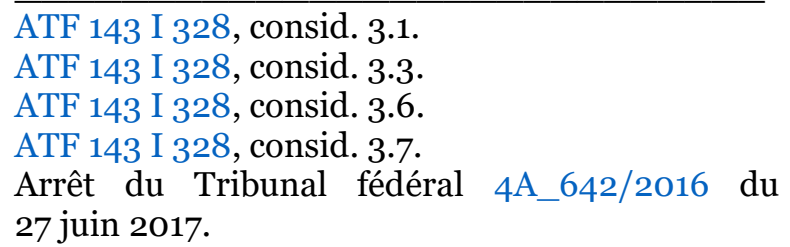

l'art. $812 \mathrm{CO}$ (qui reprend les principes de l'art. $717 \mathrm{CO}$ ), les membres de l'organe de gestion ont l'obligation de prendre leurs décisions dans l'intérêt de la société. La référence est la décision qui serait prise par une personne raisonnable et sensée se trouvant dans la même situation; ceci doit être apprécié en fonction de l'état des connaissances de la personne et du droit à l'époque des faits en question ${ }^{62}$.

65 Dans le cas d'espèce, la Haute Cour conclut que B, dans sa position d'administrateur unique de C Sàrl, a respecté son devoir de diligence lorsqu'il a décidé de rembourser la dette vis-à-vis de D SA à l'aide du prêt de A. Ce prêt ne servait pas essentiellement à investir pour assurer un rendement, mais permettait à C Sàrl de réaliser le projet commun $\mathrm{H}$. Le TF estime que cet investissement était conforme au but social et qu'il était donc dans l'intérêt de la société63. Partant, le recours est rejeté64.

\section{c) Responsabilité des personnes s'occupant de la gestion d'une Sàrl faillie ${ }^{65}$}

66 Faits : U. Sàrl, fondée par F.Z et son époux H.Z, est une société active dans le domaine immobilier, qui tombe en faillite le 26 janvier 2009. Après s'être fait céder les droits de la masse par l'office des faillites, X SA, créancière de U Sàrl, ouvre action le 23 février 2012 contre les associés

62 Arrêt du Tribunal fédéral 4A_642/2016 du 27 juin 2017, consid. 2.1.

63 Arrêt du Tribunal fédéral 4A_642/2016 du 27 juin 2017, consid. 2.5.

64 Arrêt du Tribunal fédéral 4A_642/2016 du 27 juin 2017 , consid. 3 .

65 Arrêt du Tribunal fédéral 4A_77/2017 du 26 juillet 2017. 
F.Z et H.Z en raison de leur responsabilité dans la gestion de la société faillie.

Droit : Le TF retient une mauvaise application de l'art. 827 cum 754 CO. Contrairement au jugement de deuxième instance, le TF identifie deux prélèvements privés injustifiés qui ont engendrés un dommage à la société, donnant droit à réparation au sens de l'art. 754 al. 1 CO. Ces prélèvements ont été débités après l'ouverture de la procédure de faillite, sans promesse de remboursement. Les défendeurs sont, par conséquent, devenus débiteurs de dommages-intérêts vis-à-vis de U Sàrl suite à la diminution de l'actif social, ce qui a causé un dommage à la société66. Le TF annule le jugement d'appel et renvoie la cause au Tribunal cantonal ${ }^{67}$.

\section{Doctrine}

\section{Ouvrages généraux}

68 Baker McKenzie, Zürich (édit.), Entwicklungen im schweizerischen Wirtschaftsrecht 2016/2017, Berne 2017; Urs P. Gnos/Dominik Hohler, Gesellschaftsrecht, Entwicklungen 2016, Berne 2017 ; Peter V. Kunz/Florian S. Jörg/Olivier Arter (édits), Entwicklungen im Gesellschaftsrecht XII, Berne 2017; Bernhard Madörin, Der KMU-Verwaltungsrat, $2^{\text {ème }}$ éd., Berne 2017; Pascal Montavon/ Michael Montavon/Rémy Bucheler/ Alban Matthey/Ivan Jabbour/Jeremy Reichlin, Abrégé de droit commercial, 6ème éd., Genève/Zurich/Bâle 2017; Karin Müller, Corporate Social Responsibility: Politisches Engagement von Unter-

66 Arrêt du Tribunal fédéral 4A_77/2017 du 26 juillet 2017, consid. 4.

67 Arrêt du Tribunal fédéral 4A_77/2017 du 26 juillet 2017 , consid. 5 . nehmen, Bâle 2017; Thomas Sprecher, Sanierung und Insolvenz von Unternehmen VIII : Immobilien und Insolvenz, Berne 2017; Pierre Tercier/Marc Amstutz/Rita Trigo-Trindade (édits), Commentaire romand : Code des obligations II, Bâle 2017; Florent Thouvenin, Der Kooperationsvertrag, Kooperationen im Grenzbereich von Vertrags- und Gesellschaftsrecht, Berne 2017; Rolf $\mathrm{H}$. Weber/Walter A. Stoffel/Jean-Luc Chenaux/Rolf Sethe (édits), Aktuelle Herausforderungen des Gesellschafts- und Finanzmarktrechts, Festschrift für Hans Caspar von der Crone zum 60. Geburtstag, Zurich 2017.

\section{SA}

69 Mirjam Durrer, Die Pflicht des Verwaltungsrats zum integralen Risikomanagement in KMU, thèse, Zurich/SaintGall 2017; Damian K. Graf, Gesellschaftsorgane zwischen Aktienrecht und Strafrecht, Aktienrechtliche Verantwortlichkeit, Ungetreue Geschäftsbesorgung, Misswirtschaft, thèse d'habilitation, $\mathrm{Zu}$ rich/Saint-Gall 2017; Roman S. Gutzwiller, Die Einflussmöglichkeiten des Staates auf die Strategie einer Aktiengesellschaft mit staatlicher Beteiligung, thèse (Schweizer Schriften zum Handels- und Wirtschaftsrecht 339), Zurich 2017 ; Christian Hachmann, Kriterium der Zahlungsunfähigkeit in der aktienrechtlichen Sanierung, thèse (Schweizer Schriften zum Handels- und Wirtschaftsrecht 336), Zurich 2017 ; Thomas Jutzi, Unternehmenspublizität, Grundlinien einer rechtlichen Dogmatik zur Offenlegung von unternehmensbezogenen Informationen, thèse d'habilitation, Berne 2017; Valentina Meier, Schiedsklauseln in Statuten schweizerischer Aktiengesellschaften, thèse (Schweizer Schriften zum 
Handels- und Wirtschaftsrecht 338), Zurich 2017 ; Peter Nobel, Berner Kommentar: Das Aktienrecht: Systematische Darstellung, Berne 2017; Kaspar Projer, Die Übermittlung des Aktionärswillens durch die unabhängige Stimmrechtsvertretung, thèse (Zürcher Studien zum Privatrecht 279), Zurich 2017; Nicolas Rouiller/Marc Bauen/Robert Bernet/ Colette Lasserre Rouiller, La société ano-

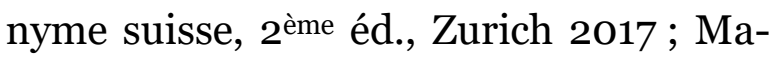
rie-Noëlle Zen-Ruffinen/Marc Bauen, Le conseil d'administration, $2^{\text {ème }}$ éd., Zurich 2017.

\section{Sàrl}

7o Christoph Feuz, Das mitgliedschaftliche Treuepflichtregime der $\mathrm{GmbH}$, thèse (Schweizer Schriften zum Handels- und Wirtschaftsrecht 340), Zurich 2017.

\section{SCoop}

71 Franco Taisch/Alexander Jungmeister/ Nadja Fabrizio, Corporate Governance von Genossenschaftsunternehmen, $\mathrm{Zu}-$ rich 2017.

\section{Raisons de commerce et registre du commerce}

72 Michael Gwelessiani/Niels Schindler, Commentaire pratique de l'Ordonnance sur le registre du commerce, $2^{\text {ème éd., }}$ Zurich 2017; Rino Siffert, Berner Kommentar : Die Geschäftsfirmen, Art. 944 956 OR, Berne 2017.

\section{LFus}

73 Rudolf Tschäni, Mergers \& Acquisitions XIX, Berne 2017; Alexander Vogel/ Christoph Heiz/Urs R. Behnisch/Andrea Sieber/Andrea Opel (édits), FusG Kom-

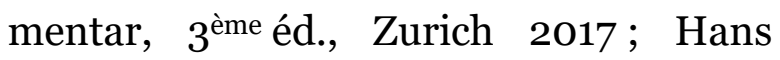
Caspar Von der Crone/Andreas Gers-
bach/Franz J. Kessler/Brigitte Von der Crone/Karin Ingber, Das Fusionsgesetz,

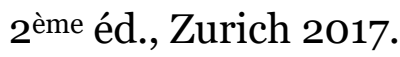

